\title{
Mapping of the interaction site of CP12 with glyceraldehyde-3-phosphate dehydrogenase from Chlamydomonas reinhardtii
}

\section{Functional consequences for glyceraldehyde-3-phosphate dehydrogenase}

\author{
Sandrine Lebreton, Simona Andreescu* ${ }^{*}$ Emmanuelle Graciet* ${ }^{* \dagger}{ }^{\dagger}$ and Brigitte Gontero \\ Institut Jacques Monod, CNRS-Universités Paris VI et Paris VII, France
}

\author{
Keywords \\ CP12; GAPDH; interaction site; intrinsically \\ unstructured protein; protein-protein \\ interactions

\section{Correspondence \\ B. Gontero, Institut Jacques Monod, UMR 7592, CNRS-Universités Paris VI et Paris VII, 2 place Jussieu, 75251 Paris cedex 5, \\ France \\ Fax: +33144275994 \\ Tel: +33144274719 \\ E-mail: meunier@ijm.jussieu.fr \\ *The authors contributed equally to this work. \\ tPresent address
California Institute of Technology, Pasadena,
CA, USA}

(Received 29 March 2006, revised 16 May 2006, accepted 25 May 2006)

doi:10.1111/j.1742-4658.2006.05342.x
The $8.5 \mathrm{kDa}$ chloroplast protein $\mathrm{CP} 12$ is essential for assembly of the phosphoribulokinase/glyceraldehyde-3-phosphate dehydrogenase (GAPDH) complex from Chlamydomonas reinhardtii. After reduction of this complex with thioredoxin, phosphoribulokinase is released but CP12 remains tightly associated with GAPDH and downregulates its NADPH-dependent activity. We show that only incubation with reduced thioredoxin and the GAPDH substrate 1,3-bisphosphoglycerate leads to dissociation of the GAPDH/ CP12 complex. Consequently, a significant twofold increase in the NADPHdependent activity of GAPDH was observed. 1,3-Bisphosphoglycerate or reduced thioredoxin alone weaken the association, causing a smaller increase in GAPDH activity. CP12 thus behaves as a negative regulator of GAPDH activity. A mutant lacking the C-terminal disulfide bridge is unable to interact with GAPDH, whereas absence of the N-terminal disulfide bridge does not prevent the association with GAPDH. Trypsin-protection experiments indicated that GAPDH may be also bound to the central $\alpha$-helix of CP12 which includes residues at position 36 (D) and 39 (E). Mutants of CP12 (D36A, E39A and E39K) but not D36K, reconstituted the GAPDH/CP12 complex. Although the dissociation constants measured by surface plasmon resonance were 2.5-75-fold higher with these mutants than with wild-type CP12 and GAPDH, they remained low. For the D36K mutation, we calculated a $7 \mathrm{kcal} \cdot \mathrm{mol}^{-1}$ destabilizing effect, which may correspond to loss of the stabilizing effect of an ionic bond for the interaction between GAPDH and CP12. It thus suggests that electrostatic forces are responsible for the interaction between GAPDH and CP12.
CP12 is a small, nuclear-encoded chloroplast protein that, in a green alga, Chlamydomonas reinhardtii, a cyanobacterium, Synechocystis PCC6803 and higher plant chloroplasts, forms part of a core complex consisting of phosphoribulokinase (PRK), glyceraldehyde3-phosphate dehydrogenase (GAPDH) and CP12
[1-6]. When embedded within the complex, the two light-regulated enzymes, which belong to the Calvin cycle, are less active than when dissociated [4,7-10]. It has also been shown that Calvin cycle activity depends on complex dissociation, controlled by the NADPH to NADP ratio, which is directly linked to electron flux

\footnotetext{
Abbreviations

BPGA, 1,3-bisphosphoglycerate; CTE, C-terminal extension; GAPDH, glyceraldehyde-3-phosphate dehydrogenase; IAA, iodoacetamide; IUP, intrinsically unstructured proteins; PRK, phosphoribulokinase; SPR, surface plasmon resonance.
} 
in the thylakoid membranes. This NADPH-mediated reversible dissociation of the supramolecular complexes seems to be conserved in all photosynthetic organisms $[1,11]$. Dissociation upon thioredoxin reduction, another important signal [12] involved in the regulation of the Calvin cycle activity [13], has also been studied in some organisms. With the algal complex, partial dissociation is observed in vitro upon reduction by thioredoxin $[8,14]$. This partial dissociation results in the release of PRK as a dimer, whereas GAPDH remains strongly associated with $\mathrm{CP} 12$ and forms a subcomplex of one tetramer of GAPDH with one monomer of CP12 [2,10]. However, in Arabidopsis thaliana, full dissociation of the complex into the three components has been observed under several conditions in vitro, and in no case, did dissociation of the ternary complex lead to a binary complex [15]. In this organism, the effect of CP12 on GAPDH activity was negligible [15], in contrast to the inhibition observed in C. reinhardtii [10]. Analysis in each organism of these association-dissociation processes linked to changes in activities of the enzymes is thus of crucial importance to find a general mode of light regulation of the Calvin cycle in all photosynthetic organisms.

GAPDH is composed of four identical GapA subunits in C. reinhardtii, whereas in higher plants there are two forms of chloroplast GAPDH which give rise to an $A_{x} B_{x}$ quaternary structure formed by GapA and GapB subunits in equal stoichiometric ratios [16-18]. The GapB subunit presents a C-terminal extension (CTE) compared with the GapA subunit (30 amino acid residues longer). This CTE is involved in kinetic regulation of the enzyme and, in particular, inhibition of GAPDH in the dark has been linked to the presence of a disulfide bridge in this CTE. It can also induce the aggregation of $\mathrm{A}_{2} \mathrm{~B}_{2}$ GAPDH into higher molecular mass polymers such as $\mathrm{A}_{8} \mathrm{~B}_{8}$ [19-21]. It has previously been shown that reduced thioredoxin and 1,3-bisphosphoglycerate (BPGA) positively regulate the NADPH-dependent activity of the $A_{2} B_{2}$ isoform $[20,22,23]$ via the CTE of the GapB subunit. This CTE seems to play a role as a kind of latch that hinders $\mathrm{NADP}(\mathrm{H})$ getting into its binding site. BPGA and thioredoxin move this CTE and thereby, NADPH-dependent activity increases [24]. CP12 shares sequence similarities with the CTE of GapB subunit [25] and thus may behave as the C-terminus extension of GapB subunits. It may also act similarly for the regulation of the $\mathrm{A}_{4}$ GAPDH of C. reinhardtii. It has also been shown that a gene-fusion event between an ancestral GAPDH and CP12 may have led to evolution of the GapB subunit [26].
In addition, we have previously shown that $\mathrm{CP} 12$ is essential for PRK/GAPDH/CP12 complex assembly and shares some physicochemical properties with intrinsically unstructured proteins (IUP) [2]. These proteins are more 'adaptative', leading to advantages in the regulation of various physiological processes and in binding diverse ligands. IUP play roles in cell-cycle control, signal transduction, transcriptional and translational regulation, as well as in macromolecular complexes [27-31]. They may be classified, depending on their functional role, as effectors that modulate the activity of a partner molecule, assemblers that act as a linker, or scavengers that store small ligands [32]. As a consequence, this novel class of proteins has 'come of age' and their recognition site, characterization and classification are one of the most challenging undertakings of structural biology in the years ahead. The first major stumbling block encountered by the researcher interested in characterizing protein-protein interactions is, nonetheless, that of having access to enough purified stable proteins. In that respect, the algal GAPDH/CP12 model is a useful tool.

In order to gain further insights into algal CP12 when associated with GAPDH, we have, for the first time, mapped its interaction site with GAPDH. For that purpose, we used proteolysis experiments coupled to MS analysis of the generated fragments to detect which regions of $\mathrm{CP} 12$ were protected upon its association with GAPDH, in combination with site-directed mutagenesis. Furthermore, to better grasp the role of CP12 as a possible effector that modulates C. reinhardtii GAPDH $\left(\mathrm{A}_{4}\right)$ activity, we measured its influence on the NADPH-dependent activity of GAPDH in the presence of BPGA and/or thioredoxin. Conditions leading to dissociation of the GAPDH/CP12 complex in the alga $C$. reinhardtii, are for deciphered.

\section{Results}

\section{Effect of CP12 on GAPDH activity}

In higher plants, BPGA and thioredoxin act on the NADPH-dependent activity of GAPDH via the CTE of the GapB subunit. In order to better understand the role of algal CP12 on the activity of GAPDH composed only of GapA subunits, GAPDH/CP12 (native GAPDH purified from C. reinhardtii cells) [10], was incubated with BPGA and/or thioredoxin for $1 \mathrm{~h}$ at $30{ }^{\circ} \mathrm{C}$ and its NADPH-dependent activity was measured (Table 1). Some changes in the catalytic rate constant were seen when native GAPDH was incubated with $10 \mu \mathrm{M}$ reduced thioredoxin or $160 \mu \mathrm{M}$ BPGA alone, as the activity increased by 39 and $33 \%$, 
Table 1. NADPH-dependent activity of GAPDH and redox state of CP12 in the GAPDH/CP12 complex. After incubation with BPGA and/or thioredoxin (Td) the GAPDH activity was measured and the redox states of CP12 determined by IAA alkylation and MS analysis as described in Experimental procedures. ND, not determined.

\begin{tabular}{|c|c|c|c|c|c|c|}
\hline Incubation mixture & Control & $\begin{array}{l}\text { BPGA } \\
(3 \mu \mathrm{M})\end{array}$ & $\begin{array}{l}\text { BPGA } \\
(160 \mu \mathrm{M})\end{array}$ & $\begin{array}{l}\text { Td } \\
(10 \mu \mathrm{M})\end{array}$ & $\begin{array}{l}\text { Td }(10 \mu \mathrm{M}) \\
\text { BPGA }(3 \mu \mathrm{M})\end{array}$ & $\begin{array}{l}\text { Td }(10 \mu \mathrm{M}) \\
\text { BPGA }(160 \mu \mathrm{M})\end{array}$ \\
\hline $\begin{array}{l}\text { Catalytic rate constant } \\
\text { of } \mathrm{GAPDH}\left(\mathrm{s}^{-1}\right)\end{array}$ & $238 \pm 12$ & $289 \pm 21$ & $316 \pm 38$ & $330 \pm 24$ & $390 \pm 19$ & $426 \pm 28$ \\
\hline $\begin{array}{l}\text { Mass increment of CP12 } \\
\text { after IAA treatment (Da) }\end{array}$ & 0 & 0 & 0 & 114 & ND & 229 \\
\hline Free sulfhydryl group on CP12 & 0 & 0 & 0 & 2 & ND & 4 \\
\hline
\end{tabular}

respectively. However, when GAPDH was simultaneously incubated with reduced thioredoxin and $160 \mu \mathrm{M}$ BPGA, the catalytic rate constant was twice that of untreated GAPDH. When the BPGA concentration added to thioredoxin was decreased from 160 to $3 \mu \mathrm{M}$, activation remained but to a lesser extent. By contrast, the catalytic rate constant of recombinant GAPDH (i.e. GAPDH without CP12) remained constant when this enzyme was incubated with reduced thioredoxin and BPGA. The value of this rate constant $\left(430 \mathrm{~s}^{-1}\right)$, is equal to that obtained with fully dissociated GAPDH/CP12 complex.

In order to link these effects on the activity of GAPDH to the integrity of the native GAPDH, native electrophoreses followed by immunoblot analysis were performed. GAPDH was always found to be associated with CP12, except upon incubation with both reduced thioredoxin and BPGA (Fig. 1). As a result of the incubation with BPGA or reduced thioredoxin

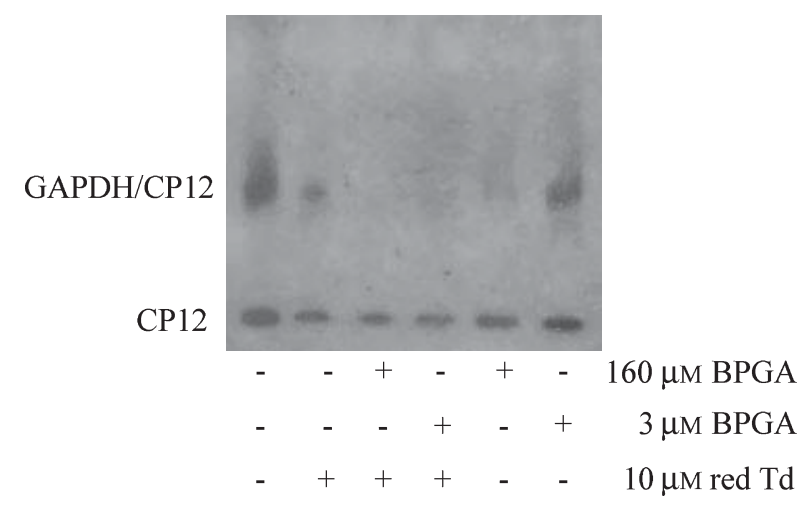

Fig. 1. Immunoblots of GAPDH/CP12 complex. The integrity of the GAPDH/CP12 complex in different incubation mixtures was checked by immunoblot. Purified native GAPDH/CP12 complex was loaded after no treatment or after incubation for $1 \mathrm{~h}$ at $30{ }^{\circ} \mathrm{C}$ with either reduced thioredoxin (red Td) or BPGA or both as indicated. Proteins $(0.76 \mu \mathrm{g}$ total corresponding to $\sim 0.04 \mu \mathrm{g}$ of $\mathrm{CP} 12)$ were separated on a native $4-15 \%$ gradient gel, transferred to a nitrocellulose membrane and revealed with antibodies raised against CP12. alone, the interaction between GAPDH and CP12 was only weakened as the proportion of GAPDH/CP12 complex decreased, but was still detected (Fig. 1).

Because CP12 is a redox-sensitive protein that possesses two disulfide bridges required for the assembly pathway of supramolecular complexes made up of GAPDH, CP12 and PRK [2,33], we analysed the redox states of CP12 (no, 2 or 4 sulfhydryl groups) in the presence of BPGA and/or thioredoxin within the GAPDH/CP12 complex. Iodoacetamide (IAA) was added to each incubation mixture described above. The degree of alkylation was monitored using MS; for each IAA molecule bound to a sulfhydryl group, a mass increment of $57.2 \mathrm{Da}$ was expected. When IAA was added to the control mixture, i.e. native GAPDH/CP12 complex, no mass increment of CP12 molecular mass was observed, indicating that no IAA molecule was bound, and that $\mathrm{CP} 12$ bound to GAPDH was fully oxidized. It has been shown that in the oxidized state, algal $\mathrm{CP} 12$ has its four cysteine residues involved into two disulfide bridges, one between cysteine residues 23 and 31, and the other between cysteine residues 66 and 75 [34]. When native GAPDH/CP12 was incubated with BPGA, again no mass increment was observed. After incubation with reduced thioredoxin, only one disulfide bridge was broken as shown by a mass increment of $\sim 114 \mathrm{Da}$. Finally, when the GAPDH/CP12 complex was incubated simultaneously with reduced thioredoxin and BPGA, four molecules of IAA were bound to CP12 (mass increment of $229 \mathrm{Da}$ ) indicating that CP12 was completely reduced. The redox states were thus linked to the integrity of the GAPDH/CP12 complex (Table 1).

In order to determine which disulfide bridge was first broken, trypsin was added to each incubation mixture previously treated with IAA. After $30 \mathrm{~min}$ at $37^{\circ} \mathrm{C}$, the proteolytic fragments were analysed by MS. A peak corresponding to the C-terminus of CP12 (2999 Da, residues 54-80, numbering from CP12 sequence without the His-tag) including residues 
Table 2. Main fragments of CP12, purified with its His-tag, obtained after trypsin digestion. The numbers of the first and last residue of each fragment are indicated in brackets. The correspondence with the sequence deleted of its Hig-tag is indicated in italics. The monoisotopic masses giving peptide masses as $\left[\mathrm{M}+\mathrm{H}^{+}\right.$that are reported are calculated from $\mathrm{CP} 12$ sequence. The asterisk represents mass obtained when the Cys residues are involved in a disulfide bridge. Residues given in bold correspond to those belonging to the His-Tag.

\begin{tabular}{|c|c|}
\hline & Mass (Da) (Residue number) \\
\hline \multicolumn{2}{|l|}{ Not digested CP12 } \\
\hline $\begin{array}{l}\text { HHHHHHHHHHSSGHIEGRHMSGQPAVDLNKKVQDAVKEAEDACAKGTSADCAV } \\
\text { AWDTVEELSAAVSHKKDAVK ADVTLTDPLEAFCKDAPDADECRVYED } \\
\text { Fragments obtained without missed cleavages }\end{array}$ & $10927(1-100)$ \\
\hline НHНHНHHHHHSSGHIEGR (His-tag) & $2270(1-18)$ \\
\hline GTSADCAVAWDTVEELSAAVSHK & $2347(46-68)(26-48)$ \\
\hline ADVTLTDPLEAFCK & $1522(74-87)(54-67)$ \\
\hline DAPDADECR & $991(88-96)(68-76)$ \\
\hline VYED & $525(97-100)(77-80)$ \\
\hline \multicolumn{2}{|l|}{ Fragments with one or more missed cleavages } \\
\hline HMSGQPAVDLNKKVODAVKEAEDACAKGTSADCAVAWDTVEELSAAVSHKK & $5338(19-69)$ \\
\hline KVODAVKEAEDACAKGTSADCAVAWDTVEELSAAVSHKK & $4060(31-69)(11-49)$ \\
\hline VODAVKEAEDACAKGTSADCAVAWDTVEELSAAVSHKK & 3932 (32-69) (12-49) \\
\hline ADVTLTDPLEAFCKDAPDADECRVYED & $2999 *, 3001(74-100)(54-80)$ \\
\hline ADVTLTDPLEAFCKDAPDADECR & $2493 *, 2495(74-96)(54-76)$ \\
\hline GTSADCAVAWDTVEELSAAVSHKK & $2475(49-69)(26-49)$ \\
\hline
\end{tabular}

C66 and C75 was found (Table 2). This peptide with no IAA molecule bound was detected indicating the presence of a disulfide bridge between these residues whether CP12/GAPDH complex was incubated with BPGA or with reduced thioredoxin alone, suggesting that this bridge was inaccessible to thioredoxin. After simultaneous incubation of the complex with BPGA and reduced thioredoxin, this peak at 2999 Da disappeared, indicating full reduction followed by complete digestion of the C-terminus of CP12 (data not shown).

As a control, we checked that thioredoxin alone was sufficient to fully reduce the recombinant CP12 in the absence of GAPDH, as evidenced by the presence of four bound IAA molecules on this form (data not shown). This indicates that the inaccessibility of the C-terminus of $\mathrm{CP} 12$ to thioredoxin in the GAPDH/CP12 complex is specifically due to the interaction of CP12 with GAPDH.

\section{Impact of cysteine residues of CP12 on the reconstitution of the subcomplex GAPDH/CP12}

As disulfide bridges on $\mathrm{CP} 12$ are required in PRK/GAPDH complex formation, reconstitution experiments were performed in vitro to check whether different cysteine mutants of CP12 were able to interact with GAPDH, as did the wild-type CP12. The mutants were designed in order to replace the cysteine residue at either position 23 or position 66 by a serine residue. The rationale behind the choice of these muta- tions was to independently disrupt either the $\mathrm{N}$ - or the C-terminal disulfide bridge. Reconstitution experiments were performed using equimolar proportions of each mutant of $\mathrm{CP} 12$ and recombinant GAPDH, as described previously [10]. After incubation for $1 \mathrm{~h}$ at $30{ }^{\circ} \mathrm{C}$, native PAGE was performed followed by immunoblot. A band recognized by both anti-CP12 and anti-GAPDH IgG appeared with the wild-type CP12 (as expected) and with the C23S mutant (Fig. 2), but not with the C66S mutant, indicating that the latter was unable to reconstitute the GAPDH/CP12 complex in vitro.

The interaction was further characterized by surface plasmon resonance (SPR; BiaCore, Uppsala, Sweden) as previously carried out with wild-type CP12 [2]. Again, no binding of C66S mutant on GAPDH was detected, while the calculated dissociation constants $\left(K_{\mathrm{d}}\right)$ between $\mathrm{C} 23 \mathrm{~S}$ mutant and GAPDH, and between wild-type CP12 and GAPDH were 0.33 and $0.4 \mathrm{~nm}$, respectively (data not shown). Wild-type CP12 and the C23S mutant thus seem to interact in the same way with recombinant GAPDH, whereas mutation of the cysteine residue at position 66 prevents interaction of CP12 with GAPDH.

\section{Identification of the interaction site between CP12 and GAPDH}

In order to identify the interaction site between $\mathrm{CP} 12$ and GAPDH, CP12, alone or in the presence of GAPDH, was digested by trypsin as a function of 


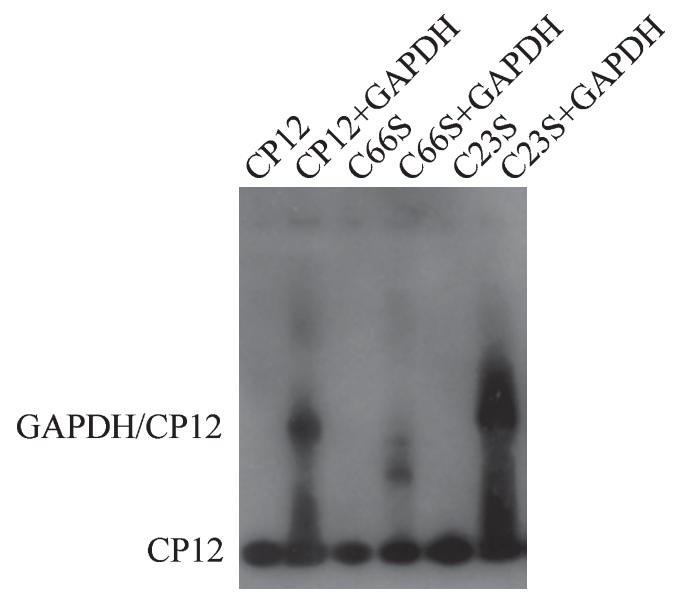

Fig. 2. Western blot analysis of the in vitro reconstitution of GAPDH/CP12 complex. GAPDH and CP12 were mixed in a molar ratio of $1: 1$. After $1 \mathrm{~h}$ at $30^{\circ} \mathrm{C}$, proteins were separated on a native $4-15 \%$ gradient gel, transferred to a nitrocellulose membrane and revealed with antibodies raised against CP12. Wild-type or mutants of CP12 $(0.03 \mathrm{nmol})$ were mixed with $0.03 \mathrm{nmol}$ of GAPDH. In each lane, same amount $(0.033 \mu \mathrm{g})$ of CP12 either alone or mixed with GAPDH was loaded. No band was revealed with recombinant GAPDH alone.

time. The resulting proteolytic fragments were then identified using MALDI-TOF MS.

After 5 min incubation with trypsin, proteolysis of recombinant wild-type $\mathrm{CP} 12$, purified with its histidine tag, generated three major fragments (Fig. 3A, Table 2$)$, a first peak ( $\sim 2270 \mathrm{Da})$ corresponding to the His-tag with one nickel bound, and two other peaks ( $\sim 2493$ and $\sim 2999 \mathrm{Da})$ matching fragments of the Cterminus of CP12 (residues 54-76 and 54-80, respectively). The numbering is always given from the CP12 sequence without the His-tag.). These two fragments contain two cysteine residues that are involved in a disulfide bridge. Indeed, when CP12 previously digested by trypsin for $5 \mathrm{~min}$ was reduced with $30 \mathrm{~mm}$ dithiothreitol for $1 \mathrm{~h}$, the same spectrum as in Fig. 3A was obtained, except that the mass of the peaks at 2999 and $2493 \mathrm{Da}$ increased by $2 \mathrm{Da}$ (3001 and $2495 \mathrm{Da}$, respectively). The two cysteine residues (C66 and C75) were thus reduced after dithiothreitol treatment. By contrast, if reduction was performed prior to digestion, the two peaks at 3001 and $2495 \mathrm{Da}$ were no longer present and were replaced, instead, by three peaks at 1522 (residues 54-67), 991 (residues 68-76) and $525 \mathrm{Da}$ (residues 77-80), corresponding to full digestion of the C-terminus of CP12, compared with partial digestion of this region with oxidized CP12 (peaks at $\sim 2493$ and $2999 \mathrm{Da}$ ) (Table 2). Thus, when CP12 is oxidized, cysteine residues 66 and 75 form a disulfide
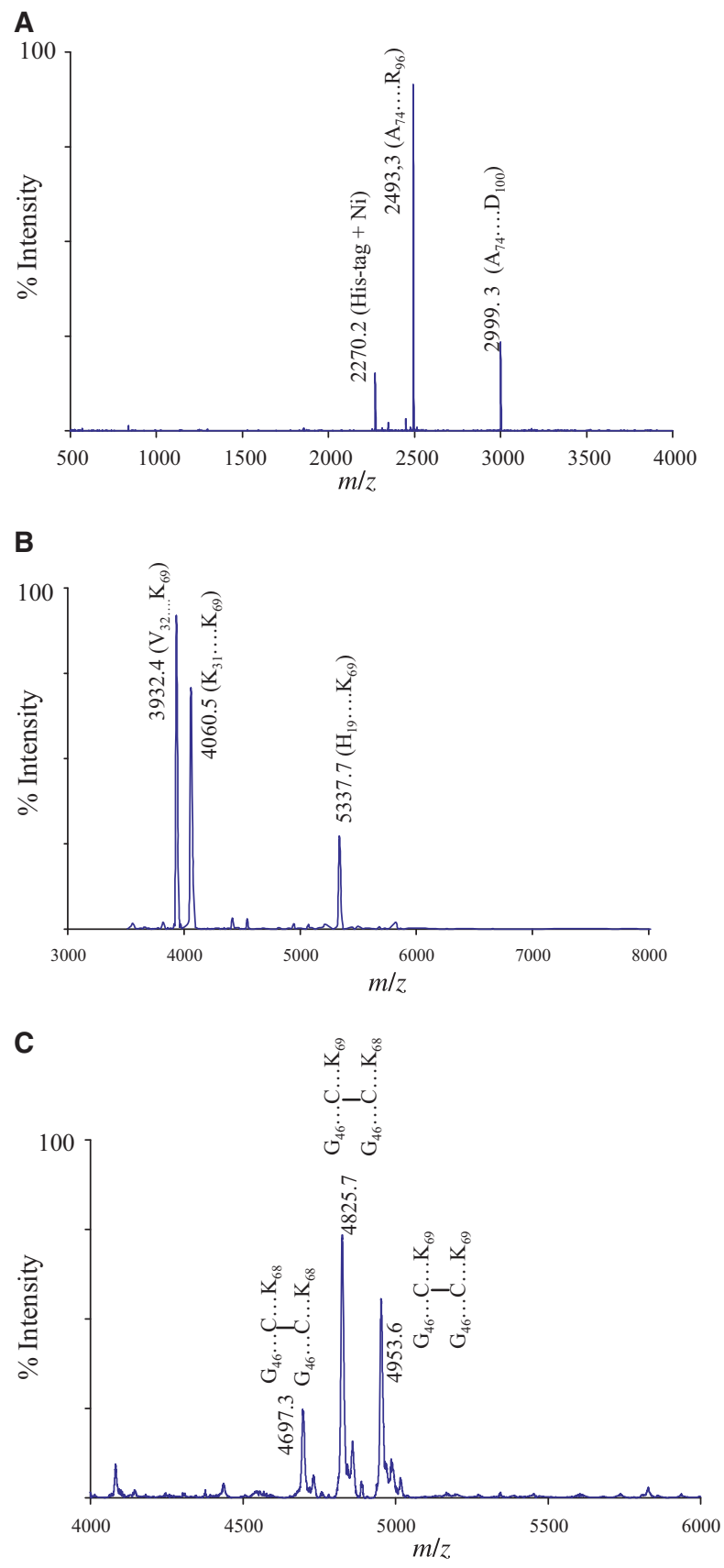

Fig. 3. Proteolytic profile of wild-type CP12. CP12 purified with its histidine tag $(2 \mu \mathrm{g})$ was incubated with trypsin (1:200 (tryp$\sin / \mathrm{CP} 12 \mathrm{w} / \mathrm{w})$ at $37{ }^{\circ} \mathrm{C}$ for $5 \mathrm{~min}(\mathrm{~A}, \mathrm{~B})$ or $30 \mathrm{~min}(\mathrm{C})$. Fragments of low $(<3000 \mathrm{Da})$ or high (>3000 Da) mass are shown, respectively, in $(A)$ and $(B)$. Samples were desalted on $C_{18}$ zip-tip and analysed by MS. The sequences of the fragments corresponding to the masses are shown in brackets. Fragments corresponding to cross-link via Cys31 are found after 30 min (C). Numbering of the fragments includes the His-tag. 
bridge that hinders cleavage of two potential sites for trypsin.

Moreover, after $5 \mathrm{~min}$, trypsin digestion was not completely achieved because fragments of higher molecular mass remained (Fig. 3B): peaks at 5338 Da (residues 1-49 plus the residues HM from the His-tag), $4060 \mathrm{Da}$ (residues 11-49) and $3932 \mathrm{Da}$ (residues 1249) corresponding to 5, 4 and 3 missed cleavages (potential sites of trypsin, arginine or lysine residues that are not cleaved) in the central and $\mathrm{N}$-terminal part of CP12 were detected.

Longer incubations of oxidized CP12 with trypsin were then carried out, and the proteolytic pattern was not dramatically altered for the small fragments $\left(M_{\mathrm{r}}<3000 \mathrm{Da}\right)$. Nonetheless, fragments of high molecular mass (5338, 4060 and $3932 \mathrm{Da}$ ) corresponding to missed cleavages decreased after $10 \mathrm{~min}$ digestion (data not shown) and disappeared after $30 \mathrm{~min}$ (Fig. 3C). Instead, three fragments $(\sim 4948, \sim 4820$ and $\sim 4692 \mathrm{Da}$ ) corresponding to cross-links between fragments of the central part $(2347$ or $2475 \mathrm{Da})$ were obtained (Table 2). These three fragments disappeared after reduction as a consequence of the intermolecular disulfide rupture involving cysteine residue 31 of each monomer (data not shown). These results indicate that the disulfide bridge in the $\mathrm{N}$-terminal part of CP12 is more labile than that in the $\mathrm{C}$-terminal part. Indeed, in CP12 preparations that have not been treated with an oxidant agent, cysteines 23 and 31 may bear free sulfhydryl groups.

Recombinant CP12 and GAPDH were mixed in different molar ratios $(1: 1,1: 0.5,1: 0.1,1: 0.01)$. Two control ratios were used $(1: 0$ and $0: 1)$. The different mixtures were incubated with trypsin for $30 \mathrm{~min}$ at $37^{\circ} \mathrm{C}$ and the proteolytic profiles were analysed by MS.

Whatever the ratio used, the fragment at $2999 \mathrm{Da}$ (residues 54-80) was always detected, indicating that the lysine at position 53 was accessible to trypsin. The same result was obtained when native GAPDH/CP12 complex, purified from $C$. reinhardtii cells, was digested (data not shown).

Remarkably, in contrast to the digestion of CP12 alone, no peaks were seen at 4948, 4820 or $4692 \mathrm{Da}$ when digestion was performed on the mixtures of CP12 and GAPDH at ratios of $1: 1$ or $1: 0.5$ (Fig. 4). These values corresponded to the central part of CP12 (2347 and $2475 \mathrm{Da}$ ) covalently linked by intermolecular disulfide bridges via Cys31. A possible explanation is that digested $\mathrm{CP} 12$ alone formed intermolecular disulfide bridges via Cys31, whereas when CP12 interacts with GAPDH, the central part is inaccessible and no cross-link occurs.

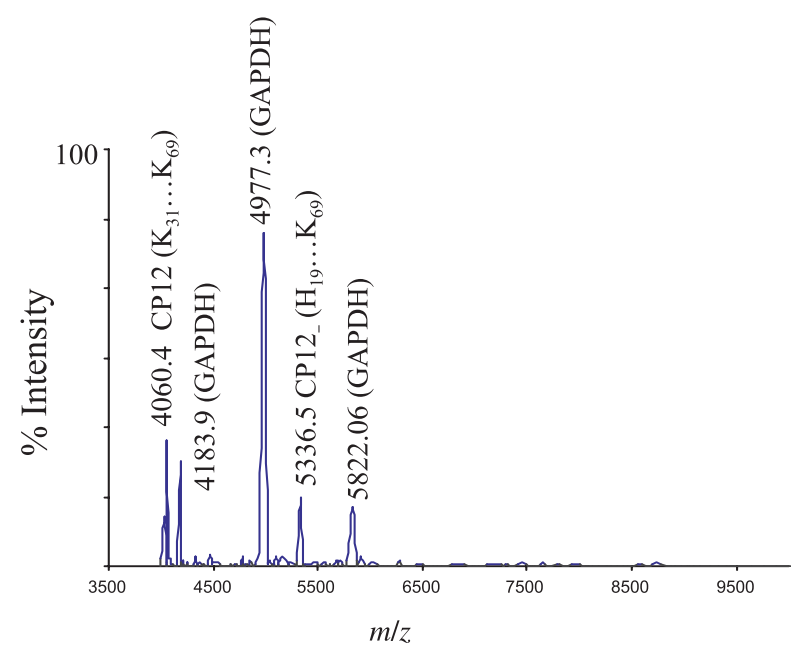

Fig. 4. Proteolytic profile of the reconstitution assay with wild-type CP12 and GAPDH in a molar ratio of $1: 1 . \mathrm{CP} 12(0.3 \mathrm{nmol})$ and GAPDH $(0.3 \mathrm{nmol})$ were mixed for $1 \mathrm{~h}$ at $30^{\circ} \mathrm{C}$, and then incubated with trypsin $(1: 200 \mathrm{w} / \mathrm{w})$ for $30 \mathrm{~min}$ at $37^{\circ} \mathrm{C}$. The sample was desalted on $\mathrm{C} 18$ zip-tip and analysed by MS. The belonging of each fragment either to GAPDH or to CP12 is indicated in brackets.

The interaction site might thus be present at the level of this fragment of molecular mass of $2347 \mathrm{Da}$ (residues 26-48), corresponding to the central part of CP12. Moreover, fragments of high molecular mass corresponding to missed cleavages [5337.5 Da (residues 1-49 plus residues HM from the His-tag) and 4060.4 Da (residues 11-49)] remained, indicating an actual protection of $\mathrm{CP} 12$ by GAPDH from trypsin digestion because these peaks were not present when CP12 alone was digested for $30 \mathrm{~min}$. The same experiment was performed with cytochrome $c$, a noninteracting CP12 protein, and no protection was observed. The same experiment was performed with the C66S mutant. No protection by GAPDH occurred. This result corroborates the data that this mutant failed to reconstitute in vitro the GAPDH/CP12 complex.

\section{Involvement of the negative charge of the residue $\mathrm{D} 36$ of $\mathrm{CP} 12$ in the interaction between CP12 and GAPDH}

In order to confirm that residues from the central part of CP12 are involved in the interaction with GAPDH, two amino acids of this region were mutated. The choice of residues Asp36 and Glu39 was based on the fact that positively charged residues of GAPDH have been shown to interact with CP12 [35]. In vitro reconstitution experiments were performed with these mutants and GAPDH. Replacing glutamate 39 with alanine or lysine did not seem to affect the formation 
$\begin{array}{llllllll}1 & 2 & 3 & 4 & 5 & 6 & 7 & 8\end{array}$

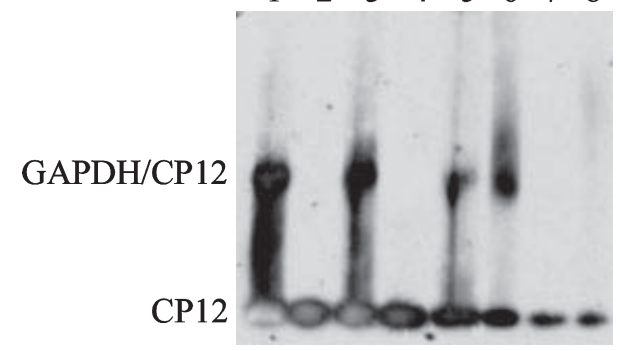

Fig. 5. In vitro reconstitution experiments of the CP12/GAPDH complex with D36 and E39 mutants of CP12. CP12 (0.03 nmol) and GAPDH $(0.03 \mathrm{nmol})$ were mixed for $1 \mathrm{~h}$ at $30^{\circ} \mathrm{C}$. Proteins were then separated on a native $4-15 \%$ gradient gel, transferred to a nitrocellulose membrane and revealed with antibodies raised against CP12. Lane 1, reconstitution mixture of wild-type CP12 and GAPDH; lane 2, wild-type CP12 alone; lane 3, reconstitution mixture of E39A mutant and GAPDH; lane 4, E39A mutant alone; lane 5, reconstitution mixture of E39K mutant with GAPDH; lane 6, reconstitution mixture of D36A mutant and GAPDH; lane 7, D36A mutant alone; and lane 8, reconstitution mixture of D36K mutant with GAPDH. The same quantity of CP12 $(0.033 \mu \mathrm{g})$ was loaded in each lane. No band was revealed with recombinant GAPDH alone. of the complex, using in vitro reconstitution experiments. Interestingly, whereas replacing aspartate 36 with alanine allowed formation of the complex, substitution of the negative charge of aspartate 36 into lysine prevented the reconstitution (Fig. 5).

The interactions between each mutant of CP12 and GAPDH were further characterized using SPR (BiaCore). The sensorgrams are reported in Fig. 6. The calculated dissociation constants $\left(K_{\mathrm{d}}\right)$ values are summarized in Table 3.

The $K_{\mathrm{d}}$ value for D36A, E39A and E39K mutants was found to be in the range 1-30 nM, compared with $0.44 \mathrm{~nm}$ for wild-type recombinant $\mathrm{CP} 12$. The $K_{\mathrm{d}}$ value for the D36K mutant increased dramatically $(\sim 45 \mu \mathrm{M})$. These values allow us to calculate the free energy of binding of GAPDH to CP12:

$$
\Delta G_{\mathrm{b}}=-R T \ln K_{\mathrm{d}}
$$

The dissociation constants of mutants CP12 with wild-type GAPDH allow calculation of the difference $\Delta \Delta G_{\mathrm{b}}$ (Eq. 2) and thus quantify the destabilization of
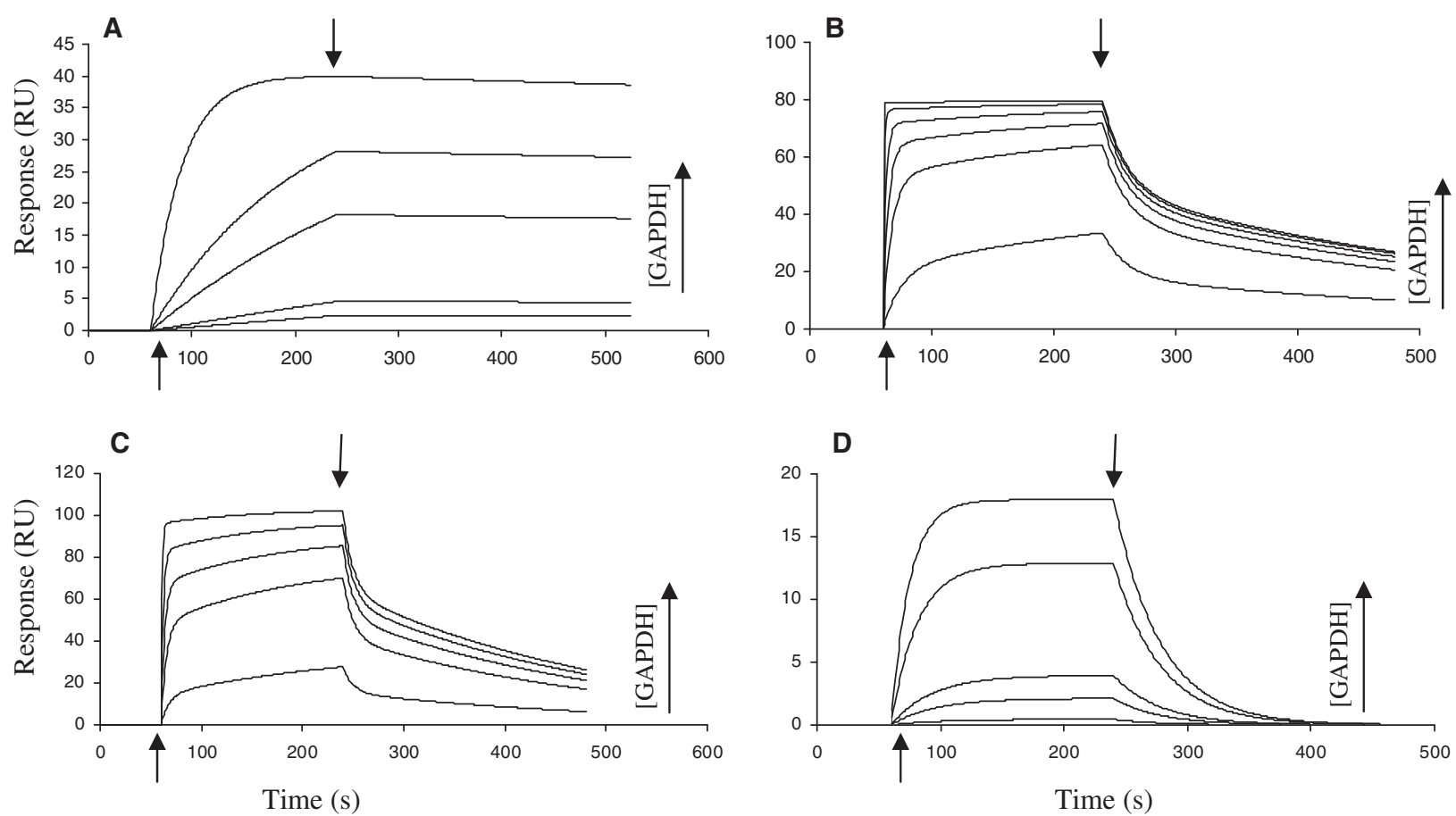

Fig. 6. Study of the interaction between CP12 mutants and GAPDH using SPR. Net sensorgrams (after subtracting the bulk refractive index) were obtained with immobilized CP12 mutants - E39A mutant (A); E39K mutant (B); D36A mutant (C) and D36K mutant (D) - using increasing concentrations of wild type recombinant GAPDH as indicated on each plot. The concentrations of wild type GAPDH were: 2.6, 5.2, 26, 52, and $260 \mathrm{~nm}(\mathrm{~A}) ; 6,30,60,120,300$, and $900 \mathrm{~nm}(\mathrm{~B}) ; 5.6,28,56,112$, and $280 \mathrm{~nm}(\mathrm{C}) ; 0.15,0.3,0.6,1$ and $1.5 \mu \mathrm{M}$ (D). The experimental data were analysed using global fitting assuming a $1: 1$ interaction. In all plots, the arrow on the left indicates the beginning of the association phase; the beginning of the dissociation phase is marked by the arrow on the right. 
Table 3. Dissociation constants and quantification of the destabilizing effect of the mutations on the interaction between mutants of CP12 and GAPDH. The dissociation constants were measured using SPR with GAPDH as the analyte and mutants or wild-type CP12 as ligands (immobilized proteins). The free energies of the association of GAPDH and CP12 were calculated according to Eqns (1) and (2).

\begin{tabular}{lcll}
\hline Ligand & $\begin{array}{l}K_{\mathrm{d}} \\
(\mathrm{nM})\end{array}$ & $\begin{array}{l}\Delta G_{\mathrm{b}} \\
\left(\mathrm{kcal} \cdot \mathrm{mol}^{-1}\right)\end{array}$ & $\begin{array}{l}\Delta G_{\mathrm{b}}^{\mathrm{WT}}-\Delta G_{\mathrm{b}}^{\text {mut }} \\
\left(\mathrm{kcal} \cdot \mathrm{mol}^{-1}\right)\end{array}$ \\
\hline Wild-type CP12 & 0.4 & -13.04 & \\
E39A CP12 & 1 & -12.48 & -0.56 \\
E39K CP12 & 13.6 & -10.91 & -2.13 \\
D36A CP12 & 30 & -10.43 & -2.61 \\
D36K CP12 & 45000 & -6.04 & -7 \\
\hline
\end{tabular}

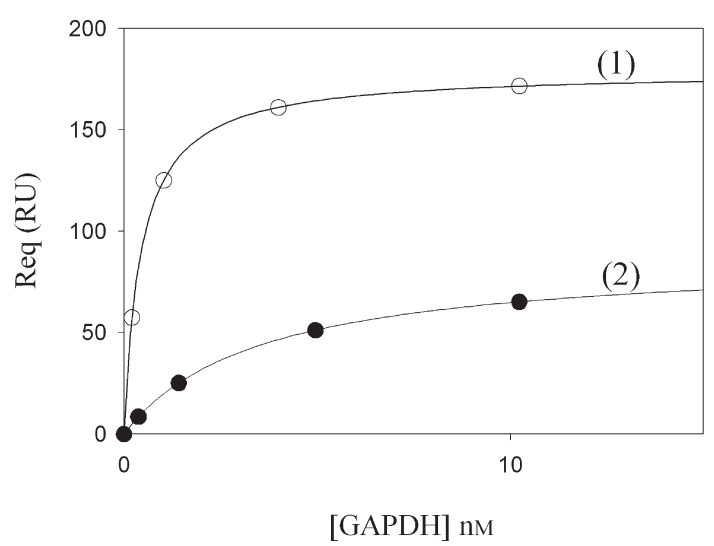

Fig. 7. Effect of salt concentration on the interaction between CP12 and GAPDH. The interaction between wild-type CP12 and GAPDH was studied using SPR with different salt concentrations. The amplitude of the plateau SPR signal $\left(R_{e q}\right)$ was plotted against the concentration of GAPDH in the presence of $0.15 \mathrm{M} \mathrm{NaCl}$ (1) or $0.32 \mathrm{M} \mathrm{NaCl}$ (2). The experimental data were fitted to Eqn (3).

the interaction between GAPDH and CP12 that could be directly linked to the point mutations introduced in CP12 (Table 3).

$$
\Delta \Delta G_{b}=\Delta G_{b}^{W T}-\Delta G_{b}^{m u t}=-R T \ln \frac{K_{d}^{W T}}{K_{d}^{m u t}}
$$

A greater effect (destabilization of $7 \mathrm{kcal} \cdot \mathrm{mol}^{-1}$ ) was observed with the D36K mutant that had previously been shown to be incapable of forming the GAPDH/ CP12 complex.

In order to screen if the interactions between CP12 and GAPDH are electrostatic in nature, SPR was performed with increasing amount of salt. The responses at equilibrium $\left(R_{\mathrm{eq}}\right)$ as a function of GAPDH concentration are reported in Fig. 7. Experimental data were fitted to the following hyperbola function:

$$
R_{e q}=\frac{R_{\max }[G A P D H]}{K_{d}+[G A P D H]}
$$

where $R_{\max }$ is the maximum analyte binding capacity in response units (RU).

At $0.65 \mathrm{M} \mathrm{NaCl}$, no binding was observed, whereas at $0.32 \mathrm{M} \mathrm{NaCl}$, a dissociation constant of $3.55 \mathrm{nM}$ was obtained, that is ninefold higher than that $(0.44 \mathrm{nM})$ obtained previously with $0.15 \mathrm{M} \mathrm{NaCl}$, 10 mм HEPES, $150 \mathrm{~mm} \mathrm{NaCl}$ (HBS).

\section{Discussion}

In cyanobacteria, it has previously been shown that the disulfide bridge on the $\mathrm{N}$-terminal part of $\mathrm{CP} 12$ is involved in binding PRK, whereas the C-terminal disulfide bridge is important for binding GAPDH $[1,6,11]$. GAPDH/CP12 complex reconstitution experiments with wild-type CP12, and two Cys mutants confirm some of these data with respect to algal GAPDH. The dissociation constants obtained by SPR between wild-type CP12 or the C23S mutant and GAPDH are almost the same $(\sim 0.3-0.4 \mathrm{nM})$. These associations are very tight, whereas no association has been detected between the C66S mutant and GAPDH. We thus propose that the C66-C75 disulfide bridge is effectively essential to the formation of the CP12-GAPDH complex. Trypsin-digestion experiments further suggest that GAPDH has to be recognized by this C-terminus but also interacts with the central part of CP12 as shown by site-directed mutagenesis and MS analysis of trypsin digestion. Indeed, a segment of $2347 \mathrm{Da}$ (residues 26-48) is protected from trypsin digestion by GAPDH. This fragment is enriched in negatively charged residues and corresponds to the central part of CP12 which has been predicted to be a $\alpha$-helix in the modelling of CP12 [36]. Remarkably, we have previously shown that substitution of residue Arg197 of C. reinhardtii GAPDH, which is located in the S-loop, with glutamate prevents formation of the GAPDH/ CP12 complex [35], and similar electrostatic interactions have been described between the CTE of the GapB subunit of GAPDH and its S-loop [15,37]. These observations together strongly suggest that the negatively charged central region of CP12 may be involved in electrostatic interactions with the positively charged residues of GAPDH S-loop [35,37,38]. We therefore mutated Asp36 and Glu39 of CP12 into Ala or Lys. To test whether the interactions of these mutants with the other partner, GAPDH were impaired, we tried to reconstitute in vitro the GAPDH/ CP12 complex. The E39A/K and D36A CP12 mutants reconstitute the GAPDH/CP12 complex. Although the 
dissociation constants measured by SPR are $\sim 3-75$ fold higher than that of wild-type recombinant GAPDH and CP12, they remain low. The D36K mutant does not reconstitute the complex, as shown by native PAGE and its dissociation constant is much higher than that of the wild-type recombinant $\mathrm{CP} 12$ ( $\sim 10^{5}$-fold). Thus, among the D36 and E39 mutants, the mutation that most destabilizes the interaction with GAPDH is the introduction of a positive charge at position 36 . The negative charge borne by the residue D36, which is located in the central part of CP12, thus seems to be required for the interaction with GAPDH. This result also suggests that Asp36 is probably exposed to the solvent whereas Glu39 may rather buried because its mutation does not strongly affect the interaction with GAPDH. It thus confirms the position of these residues within the $\mathrm{CP} 12$ structure obtained by modelling [36]. For the D36K mutation, we calculated a $7 \mathrm{kcal} \cdot \mathrm{mol}^{-1}$ destabilizing effect, which may correspond to the loss of the stabilizing effect of an ionic bond for the interaction between GAPDH and CP12. In a previous report, we mentioned the possibility of a major role of salt bridges in the interaction between GAPDH and CP12 [35]. The results presented here further support this hypothesis as we show using SPR that the interaction between $\mathrm{CP} 12$ and GAPDH is strongly affected by the presence of salt.

Our results also clearly show that two parts of CP12 are involved in the interaction with GAPDH (Fig. 8). The C-terminal disulfide bridge contributes to the interaction with GAPDH and probably to the correct folding of the central part as well. The negatively charged central region of $\mathrm{CP} 12$ is also involved in

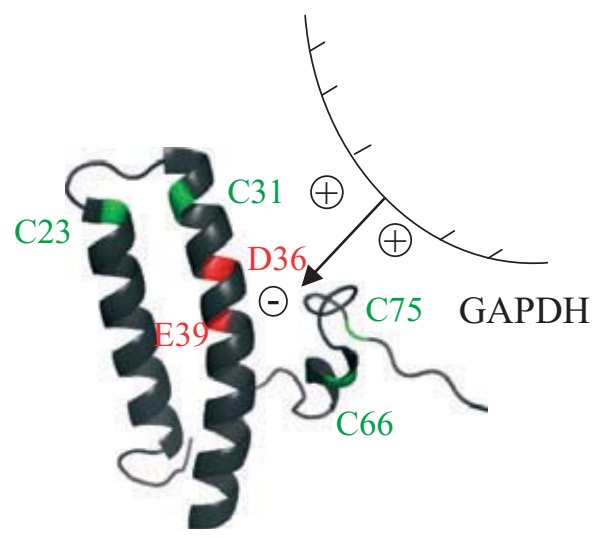

Fig. 8. Modelled structure of CP12 [36] and interaction site with GAPDH. Cysteine residues and mutated negatively charged residues are given in green and red, respectively. They are labelled. The electrostatic nature between positively charged residues located in the S-loop on GAPDH [35] and negatively charged residues on CP12 is represented by circles. stabilizing the interaction with GAPDH. The C-terminus of CP12 lacks ordered structure but is essential for the interaction with GAPDH. It is likely that some folding of this fragment occurs upon GAPDH binding. This point is a common feature of IUP [30].

In a previous report, we showed that the algal GAPDH activity was redox regulated via its interaction with PRK in the PRK/GAPDH/CP12 complex, but not when the GAPDH was free [9]. Here, we show that GAPDH activity is also modulated through its interaction with CP12, as incubation with reduced thioredoxin and BPGA together leads to dissociation of the GAPDH/CP12 subcomplex and to an increase in NADPH-dependent GAPDH activity of $\sim 80 \%$. Incubation of GAPDH/CP12 complex with either reduced thioredoxin or BPGA alone leads to partial destabilization of the complex, and to a smaller increase in GAPDH activity. Alkylation experiments show that, in the case of thioredoxin, this destabilization is generated by a partial reduction of CP12 (disruption of the $\mathrm{N}$-terminal disulfide bridge). This result corroborates our hypothesis that the C-terminus of $\mathrm{CP} 12$ is probably buried within the CP12/GAPDH complex. In addition, because incubation with BPGA also slightly destabilizes the GAPDH/CP12 complex, it is likely that BPGA, which is negatively charged, may interfere with electrostatic forces between the S-loop of GAPDH and CP12. Thioredoxin and BPGA thus have a synergistic effect that promotes release of CP12 from the GAPDH/CP12 complex. Once CP12 is released, the two disulfide bridges become accessible and may consequently be reduced by thioredoxin, as shown by the alkylation experiment. Interestingly, upon BPGA and reduced thioredoxin treatment, similar dissociation was observed with the $\mathrm{A}_{8} \mathrm{~B}_{8}$ isoform of GAPDH leading to the $\mathrm{A}_{2} \mathrm{~B}_{2}$ isoform with a higher NADPHdependent activity [39].

By analogy with the CTE of the isoform of GAPDH $[15,37]$, we hypothesize that the disulfide bridge at the C-terminus of CP12 might confer a conformation to CP12 that hinders the fine sensing of NADPH by Chlamydomonas GAPDH, when the latter is associated with $\mathrm{CP} 12$. In the model proposed by Sparla et al. on the $\mathrm{A}_{2} \mathrm{~B}_{2}$ isoform of GAPDH [24,37], formation of a disulfide bridge in the oxidized CTE prevents the interaction between the 2'-phosphate of NADPH and the side chain of Arg77 (spinach numbering, corresponds to Arg82 in C. reinhardtii GAPDH). After reduction of the CTE, Arg77 becomes free to interact with $\mathrm{NADPH}$, and consequently, NADPH-dependent activity of GAPDH increases. In the C. reinhardtii GAPDH/CP12 complex, the interaction of GAPDH Arg82 with the $2^{\prime}$-phosphate of NADPH could also be 
impaired. As soon as interactions between CP12 and GAPDH are broken by the simultaneous use of reduced thioredoxin and BPGA, this residue arginine might become accessible to NADPH, resulting in an increase of the NADPH-dependent activity.

To conclude, we have shown that CP12 is a multifunctional protein, because it acts as a linker during the assembly of the PRK/GAPDH/CP12 complex, and as a modulator of GAPDH activity by enabling fine regulation of its NADPH-dependent activity by thioredoxin and the GAPDH substrate, BPGA.

To date, mapping of the interaction site of CP12 had not studied but our results give some clues with regard to the binding of $\mathrm{CP} 12$ to GAPDH. The impact of CP12 binding on the activity of GAPDH is also further characterized.

\section{Experimental procedures}

\section{Site-directed mutagenesis}

In vitro mutagenesis was performed using QuickChange ${ }^{\mathrm{TM}}$ site-directed mutagenesis kit (Stratagene). Primers were as follows: C23s, 5'-GCTGAGGACGCTTCCGCCAAGGG TACCTCC-3'; C66s, 5'-CCCTGGAAGCTTTCTCCAAG GATGCCCCCG-3'; D36a， 5'-GCGCCGTGGCCTGGGC CACCGTTGAGGAGCTCAGCGC-3'; D36k， 5'-GCG CCGTGGCCTGGAAGACCGTTGAGGAGCTCAGC GC-3'; E39a, 5'-GCCTGGGACACCGTTGCGGAGCTC AGCGCTGC-3'; E39k, 5'-GCCTGGGACACCGTTAA GGAGCTCAGCGCTGC-3.'

All the mutations were confirmed by sequencing.

\section{Protein purification}

Recombinant wild-type CP12 with its His-tag was purified from Escherichia coli cells to apparent homogeneity, as described previously [2]. The same protocol was followed for all mutants. All the $\mathrm{CP} 12$ proteins were dialysed against $30 \mathrm{~mm}$ Tris/ $\mathrm{HCl}, 0.1 \mathrm{M} \mathrm{NaCl}, \mathrm{pH} 7.9$ and stored at $-20{ }^{\circ} \mathrm{C}$.

Recombinant and native GAPDH were purified to apparent homogeneity from $E$. coli cells and C. reinhardtii cells, respectively, as described previously [10]. Both were dialysed against $30 \mathrm{~mm}$ Tris $/ \mathrm{HCl}, 0.1 \mathrm{M} \mathrm{NaCl}, 1 \mathrm{~mm}$ EDTA, $0.1 \mathrm{~mm}$ NAD, $5 \mathrm{~mm}$ Cys $\mathrm{pH} 7.9$ and stored at $-80{ }^{\circ} \mathrm{C}$ in $10 \%$ aqueous glycerol.

Protein concentration was assayed with the Bio-Rad (Hercules, CA, USA) protein dye reagent, using BSA as a standard [40].

\section{Activity measurements}

GAPDH was incubated in $30 \mathrm{~mm}$ Tris/ $\mathrm{HCl}, 4 \mathrm{~mm}$ EDTA, $0.1 \mathrm{~mm}$ NAD pH 7.9 at $30^{\circ} \mathrm{C}$, with $160 \mu \mathrm{M}$ or
$3 \mu \mathrm{M}$ BPGA alone, or with $0.5 \mathrm{~mm}$ dithiothreitol and $10 \mu \mathrm{M}$ thioredoxin supplemented or not with 160 or $3 \mu \mathrm{M}$ BPGA. The BPGA concentration was calculated according to [39]. Aliquots were withdrawn at intervals and the activity of GAPDH was measured with NADPH as cofactor using a Pye Unicam UV2 spectrophotometer (Cambridge, UK) [10].

\section{In vitro GAPDH/CP12 complex reconstitution}

Wild-type or mutants of CP12 $(0.03 \mathrm{nmol})$ were mixed with GAPDH $(0.03 \mathrm{nmol})$ in $30 \mathrm{~mm}$ Tris $/ \mathrm{HCl}, 0.1 \mathrm{M} \mathrm{NaCl}$, $1 \mathrm{~mm}$ EDTA, $0.1 \mathrm{~mm}$ NAD, $5 \mathrm{~mm}$ Cys $\mathrm{pH} 7.9$ for $1 \mathrm{~h}$ at $30{ }^{\circ} \mathrm{C}$. The formation of the GAPDH/CP12 complex with wild-type and different mutants of CP12 were checked using native PAGE performed on $4-15 \%$ minigels using a Pharmacia Phastsystem apparatus (Pharmacia, Little Chalfont, Bucks, UK). Proteins were transferred to nitrocellulose filters $(0.45 \mu \mathrm{m}$, Schleicher and Schüll, Dassel, Germany) by passive diffusion for $16 \mathrm{~h}$. The filter was then immunoblotted with a rabbit antiserum directed against recombinant C. reinhardtii CP12 (1:10 000) or a rabbit antiserum directed against recombinant C. reinhardtii GAPDH (1: 100 000). Antibody binding was revealed using enhanced chemiluminescence detection system (Amersham, Little Chalfont, Bucks, UK) as described by the manufacturer.

\section{Biosensor assays}

Purified mutants of recombinant CP12 $\left(30 \mu \mathrm{g} \cdot \mathrm{mL}^{-1}\right)$ were coupled to carboxymethyl dextran-coated biosensor chip (CM5, BiaCore) following the manufacturer's instructions. We studied the interaction of wild-type recombinant GAPDH to each immobilized mutant of CP12 using HBS running buffer (BiaCore) supplemented with $0.1 \mathrm{~mm}$ NAD, $5 \mathrm{~mm}$ Cys, pH 7.9 at $20 \mu \mathrm{L} \cdot \mathrm{min}^{-1}$. Different concentrations of GAPDH were injected (analyte). The $\mathrm{NaCl}$ concentration was increased from $0.15 \mathrm{M}$ (HBS buffer) to $0.65 \mathrm{M}$ as indicated in the text. The analyte interacts with the ligand (CP12) to give the association phase, then, the analyte begins to dissociate as soon as injection is stopped and replaced by buffer. The observed curves were fitted assuming single-phase kinetics (single-phase dissociation/association). The kinetic parameters were calculated from these fits using BIAEVALUATION software (v2.1, BiaCore).

\section{Titration of sulfhydryl groups}

Sulfhydryl groups were quantified by alkylation of the cysteine residues with IAA prepared as described previously [41]. Recombinant CP12 or GAPDH/CP12 complex from C. reinhardtii was incubated with $100 \mathrm{~mm}$ iodoacetamide 
for $1 \mathrm{~h}$ at room temperature in the dark and analysed by MALDI-TOF MS as described previously [9].

\section{Trypsin digestions}

Oxidized recombinant CP12 and reconstituted GAPDH/ CP12 complex were incubated with trypsin (Sigma, St Louis, MI, USA) in $25 \mathrm{~mm} \mathrm{NH} \mathrm{NCO}_{3}, 5 \mathrm{~mm} \mathrm{CaCl}_{2}$ buffer at $37{ }^{\circ} \mathrm{C}$ for fixed times as specified in the main text. The ratio (protein/trypsin) used was $200: 1(\mathrm{w} / \mathrm{w})$. The digestion was stopped by adding $1 \%$ trifluoroacetic acid. The samples were then desalted on zip-tip $\mathrm{C}_{18}$ and analysed by MALDI-TOF MS [10].

To digest reduced $\mathrm{CP} 12$, the protein was incubated with $30 \mathrm{~mm}$ dithiothreitol or $10 \mu \mathrm{M}$ reduced thioredoxin for $1 \mathrm{~h}$ at $30^{\circ} \mathrm{C}$. The sulfhydryl groups were then blocked by $100 \mathrm{~mm}$ IAA and the reduced CP12 was incubated with trypsin as described above.

\section{MS analysis}

Proteolytic fragments after trypsin digestion and alkylation of CP12 were analysed by MALDI-TOF MS (Voyager DE Pro mass spectrometer from Applied Biosystems, Foster City, CA, USA). All samples were prepared as described previously [10]. Residues numbering were from the sequence of CP12 plus the His-tag except when referring to cysteine residues that were numbered from the sequence of CP12 without the tag.

\section{References}

1 Wedel N \& Soll J (1998) Evolutionary conserved light regulation of Calvin cycle activity by NADPH-mediated reversible phosphoribulokinase/CP12/glyceraldehyde-3phosphate dehydrogenase complex dissociation. Proc Natl Acad Sci USA 95, 9699-9704.

2 Graciet E, Gans P, Wedel N, Lebreton S, Camadro JM \& Gontero B (2003) The small protein CP12: a protein linker for supramolecular assembly. Biochemistry $\mathbf{4 2}$, 8163-8170.

3 Scheibe R, Wedel N, Vetter S, Emmerlich V \& Sauermann SM (2002) Co-existence of two regulatory NADPglyceraldehyde 3-P dehydrogenase complexes in higher plant chloroplasts. Eur J Biochem 269, 5617-5624.

4 Graciet E, Lebreton S \& Gontero B (2004) Emergence of new regulatory mechanisms in the Benson-Calvin pathway via protein-protein interactions: a glyceraldehyde-3-phosphate dehydrogenase/CP12/phosphoribulokinase complex. J Exp Bot 55, 1245-1254.

5 Mouche F, Gontero B, Callebaut I, Mornon JP \& Boisset N (2002) Striking conformational change suspected within the phosphoribulokinase dimer induced by interaction with GAPDH. $J$ Biol Chem 277, 6743-6749.
6 Tamoi M, Miyazaki T, Fukamizo T \& Shigeoka S (2005) The Calvin cycle in cyanobacteria is regulated by $\mathrm{CP} 12$ via the $\mathrm{NAD}(\mathrm{H}) / \mathrm{NADP}(\mathrm{H})$ ratio under light/ dark conditions. Plant J 42, 504-513.

7 Lebreton S, Gontero B, Avilan L \& Ricard J (1997) Memory and imprinting effects in multienzyme complexes - II. Kinetics of the bienzyme complex from Chlamydomonas reinhardtii and hysteretic activation of chloroplast oxidized phosphoribulokinase. Eur $J$ Biochem 246, 85-91.

8 Lebreton S \& Gontero B (1999) Memory and imprinting in multienzyme complexes. Evidence for information transfer from glyceraldehyde-3-phosphate dehydrogenase to phosphoribulokinase under reduced state in Chlamydomonas reinhardtii. J Biol Chem 274, 20879-20884.

9 Lebreton S, Graciet E \& Gontero B (2003) Modulation, via protein-protein interactions, of glyceraldehyde-3phosphate dehydrogenase activity through redox phosphoribulokinase regulation. J Biol Chem 278, 12078 12084.

10 Graciet E, Lebreton S, Camadro JM \& Gontero B (2003) Characterization of native and recombinant A4 glyceraldehyde 3-phosphate dehydrogenase. Eur J Biochem 270, 129-136.

11 Wedel N, Soll J \& Paap BK (1997) CP12 provides a new mode of light regulation of Calvin cycle activity in higher plants. Proc Natl Acad Sci USA 94, 1047910484 .

12 Buchanan BB \& Balmer Y (2005) Redox regulation: a broadening horizon. Annu Rev Plant Biol 56, 187-220.

13 Schurmann P \& Jacquot JP (2000) Plant thioredoxin systems revisited. Annu Rev Plant Physiol Plant Mol Biol 51, 371-400.

14 Avilan L, Gontero B, Lebreton S \& Ricard J (1997) Memory and imprinting effects in multienzyme complexes - I. Isolation, dissociation, and reassociation of a phosphoribulokinase-glyceraldehyde-3-phosphate dehydrogenase complex from Chlamydomonas reinhardtii chloroplasts. Eur J Biochem 246, 78-84.

15 Marri L, Sparla F, Pupillo P \& Trost P (2005) Co-ordinated gene expression of photosynthetic glyceraldehyde3-phosphate dehydrogenase, phosphoribulokinase, and CP12 in Arabidopsis thaliana. J Exp Bot 56, 73-80.

16 Cerff R (1979) Quaternary structure of higher plant glyceraldehyde-3-phosphate dehydrogenases. Eur $J$ Biochem 94, 243-247.

17 Cerff R \& Chambers SE (1979) Subunit structure of higher plant glyceraldehyde-3-phosphate dehydrogenases (EC 1.2.1.12 and EC 1.2.1.13). J Biol Chem 254, 60946098.

18 Ferri G, Comerio G, Iadarola P, Zapponi MC \& Speranza ML (1978) Subunit structure and activity of glyceraldehyde-3-phosphate dehydrogenase from spinach chloroplasts. Biochim Biophys Acta 522, 19-31. 
19 Baalmann E, Scheibe R, Cerff R \& Martin W (1996) Functional studies of chloroplast glyceraldehyde-3-phosphate dehydrogenase subunits $\mathrm{A}$ and $\mathrm{B}$ expressed in Escherichia coli: formation of highly active $\mathrm{A}_{4}$ and $\mathrm{B}_{4}$ homotetramers and evidence that aggregation of the $\mathrm{B}_{4}$ complex is mediated by the $\mathrm{B}$ subunit carboxy terminus. Plant Mol Biol 32, 505-513.

20 Scheibe R, Baalmann E, Backhausen JE, Rak C \& Vetter S (1996) C-Terminal truncation of spinach chloroplast NAD(P)-dependent glyceraldehyde-3-phosphate dehydrogenase prevents inactivation and reaggregation. Biochim Biophys Acta 1296, 228-234.

21 Qi J, Isupov MN, Littlechild JA \& Anderson LE (2001) Chloroplast glyceraldehyde-3-P dehydrogenase contains a single disulfide bond located in the C-terminal extension to the B-subunit. J Biol Chem 276, 35247-35252.

22 Baalmann E, Backhausen JE, Kitzmann C \& Scheibe R (1994) Regulation of NADP-dependent glyceraldehydes 3-phosphate dehydrogenase activity in spinach chloroplast. Bot Acta 107, 313-320.

23 Pupillo P \& Piccari GG (1975) The reversible depolymerization of spinach chloroplast glyceraldehyde-phosphate dehydrogenase. Eur J Biochem 51, 475-482.

24 Sparla F, Zaffagnini M, Wedel N, Scheibe R, Pupillo P \& Trost P (2005) Regulation of photosynthetic GAPDH dissected by mutants. Plant Physiol 138, 2210-2219.

25 Pohlmeyer K, Paap BK, Soll J \& Wedel N (1996) CP12: a small nuclear-encoded chloroplast protein provides novel insights into higher-plant GAPDH evolution. Plant Mol Biol 32, 969-978.

26 Petersen J, Teich R, Becker B, Cerff R \& Brinkmann H (2006) The GapA/B gene duplication marks the origin of streptophyta (charophytes and land plants). Mol Biol Evol in press.

27 Romero P, Obradovic Z, Li X, Garner EC, Brown CJ \& Dunker AK (2001) Sequence complexity of disordered protein. Proteins 42, 38-48.

28 Uversky VN (2002) Natively unfolded proteins: a point where biology waits for physics. Protein Sci 11, 739-756.

29 Fink AL (2005) Natively unfolded proteins. Curr Opin Struct Biol 15, 35-41.

30 Dyson HJ \& Wright PE (2005) Intrinsically unstructured proteins and their functions. Nat Rev Mol Cell Biol 6, 197-208.

31 Ward JJ, Sodhi JS, McGuffin LJ, Buxton BF \& Jones DT (2004) Prediction and functional analysis of native disorder in proteins from the three kingdoms of life. J Mol Biol 337, 635-645.
32 Tompa P (2005) The interplay between structure and function in intrinsically unstructured proteins. FEBS Lett 579, 3346-3354.

33 Marri L, Trost P, Pupillo P \& Sparla F (2005) Reconstitution and properties of the recombinant glyceraldehyde-3-phosphate dehydrogenase/CP12/phosphoribulokinase supramolecular complex of Arabidopsis. Plant Physiol 139, 1433-1443.

34 Delobel A, Graciet E, Andreescu S, Gontero B, Halgand F \& Laprévote O (2005) Mass spectrometric analysis of the interactions between a chloroplast protein, $\mathrm{CP} 12$ and metal ions. A possible regulatory role within a PRK/GAPDH/CP12 complex. Rapid Commun Mass Spectrom 19, 3379-3388.

35 Graciet E, Mulliert G, Lebreton S \& Gontero B (2004) Involvement of two positively charged residues of Chlamydomonas reinhardtii glyceraldehyde-3-phosphate dehydrogenase in the assembly process of a bi-enzyme complex involved in $\mathrm{CO}$ assimilation. Eur J Biochem 271, 4737-4744.

36 Gardebien F, Thangudu RR, Gontero B \& Offmann B (2006) Construction of a 3D model of CP12, a protein linker. J Mol Graph Model 25, 186-195.

37 Sparla F, Fermani S, Falini G, Zaffagnini M, Ripamonti A, Sabatino P, Pupillo P \& Trost P (2004) Coenzyme site-directed mutants of photosynthetic A(4)GAPDH show selectively reduced NADPH-dependent catalysis, similar to regulatory AB-GAPDH inhibited by oxidized thioredoxin. J Mol Biol 340, 1025-1037.

38 Sparla F, Pupillo P \& Trost P (2002) The C-terminal extension of glyceraldehyde-3-phosphate dehydrogenase subunit B acts as an autoinhibitory domain regulated by thioredoxins and nicotinamide adenine dinucleotide. J Biol Chem 277, 44946-44952.

39 Baalmann E, Backhausen JE, Rak C, Vetter S \& Scheibe R (1995) Reductive modification and nonreductive activation of purified spinach chloroplast NADPdependent glyceraldehyde-3-phosphate dehydrogenase. Arch Biochem Biophys 324, 201-208.

40 Bradford MM (1976) A rapid and sensitive method for the quantitation of microgram quantities of protein utilizing the principle of protein-dye binding. Anal Biochem 72, 248-254.

41 Gray WR (1993) Disulfide structures of highly bridged peptides: a new strategy for analysis. Protein Sci $\mathbf{2}$, 1732-1748. 